\title{
Un modelo de definiciones terminográficas para un glosario de documentos litúrgicos virreinales de México
}

\section{A terminographical definition model for a glossary of Mexican vice regal liturgical documents}

GACCESO ABIERTO / OPEN ACCESS

Cita: Molina Salas, Claudio, Ruiz Caballero, Antonio y Hernández Pech, Salvador (2020). Un modelo de definiciones terminográficas para un glosario de documentos litúrgicos virreinales de México. Textos en Proceso, 6(1), 96-117. https://doi.org/10.17710/tep.2020.6. 1.6molinaruizhernandez

Editores: Esperanza Alcaide Lara, Universidad de Sevilla, Ana Pano Alamán, Università di Bologna

Recibido: 27-04-2020

Aceptado: 25-05-2020

Conflicto de intereses: Los autores han declarado que no poseen conflicto de intereses.

Copyright: (c) C. Molina Salas, A. Ruiz Caballero, S. Hernández Pech. Esta obra está bajo licencia Creative Commons Reconocimiento 4.0.

\section{Claudio Molina Salinas (i) Antonio Ruiz Caballero Salvador Hernández Pech}

${ }^{1}$ Instituto de Investigaciones Estéticas, Universidad Nacional Autónoma de México, México

claudio.molina.salinas@comunidad.unam.mx

2 Escuela Nacional de Antropología e Historia Universidad Nacional Autónoma de México, México

antonio.ruiz.cab@gmail.com

${ }^{3}$ Colegio de Bibliotecología, Universidad Nacional Autónoma de México

pireracua.pech@gmail.com

\section{Resumen}

Considerando que se describen muchos tipos de definiciones en la literatura tocante a la terminografía, incluso comunes a la lexicografía, y que, potencialmente, cualquiera de estos modelos podría usarse para la construcción de definiciones de conceptos del área de la música litúrgica, en el presente trabajo de investigación se pretende ahondar sobre cuáles son los tipos más frecuentes y cuáles son sus posibles combinaciones; esto, para ofrecer un modelo útil a redactores que colaboren en proyectos académicos en los que se defina léxico, en principio, afines al área de la música litúrgica. Luego de formalizar un marco conceptual adecuado para este artículo y la problemática abordada en él, se plantean los pasos seguidos en un estudio de caso en el que se evalúan y clasifican las definiciones hechas por algunos especialistas. Tomando en cuenta esta base de análisis, se concreta una propuesta que, dentro del área de la música litúrgica, funciona como un modelo definitorio efectivo, para esta primera etapa del proyecto. El modelo de definición compuesta propuesta se constituye a partir de un tipo principal de definición (definición analítica) complementada por definición funcional, una extensional, una enciclopédica y una definición sinonímica. Por último, se explica cómo se 
determinó esta propuesta, cómo se puso a prueba el modelo y cómo se valoró la efectividad de este y se ilustran las adecuaciones para su posible implementación.

Palabras clave: definición terminográfica, modelo de definición terminológica, tipos de definiciones, redacción de definiciones, terminología de la música litúrgica.

\begin{abstract}
Given that many different types of definitions are described in the literature related to terminography, even those common to lexicography, and also that, potentially, any of these models could be used to construct definitions of concepts of the liturgical music area, this work aims at deepening in which the most frequent types are, as well as their possible combinations, in order to offer a useful model to editors who collaborate in academic projects in which lexicon is defined, an which are, in principle, related to the area of liturgical music. After formalizing a suitable conceptual framework for this article and the addressed problematic, the steps that were followed in this work in a case study which evaluates and classifies the definitions done by some specialists are presented. Taking this analysis foundation into account, a proposal is set which, within the area of liturgical music, works as an effective definitory model. The proposed definition model is constructed from a main type of definition (aristotelic definition) complemented by a functional definition, an extensional, an encyclopedic and a synonym definition. Lastly, an explanation is provided on how the model was put to the test and how its effectiveness was evaluated, as well as the adaptations for its possible implementation.
\end{abstract}

Keywords: terminographic definition, terminological definition model, types of definitions, definition writing, terminology of liturgic music.

\title{
1. Introducción
}

En un mundo cambiante, en el que la demanda de datos abiertos es una inminente realidad, es muy frecuente encontrar iniciativas gubernamentales (gobiernos digitales) y proyectos académicos que pretenden abrir sus repositorios de información, así como sus bases de datos, a la consulta pública.

Es en este contexto en que el Seminario de Música en la Nueva España y el México Independiente, con sede en el Instituto de Investigaciones Estéticas de la Universidad Nacional Autónoma de México, se ha convertido en una iniciativa de investigación académica que ha publicado en línea distintos documentos que constituyen una base empírica en el terreno de la investigación sobre la música litúrgica en la Nueva España (s. XVI al s. XIX).

Este seminario, en una de sus más recientes líneas de trabajo, tiene por objetivo la creación de un glosario de términos provenientes de la documentación publicada en su página web ${ }^{1}$ (herramienta de descripción terminológica) que funja como un facilitador del entendimiento de los textos recopilados, esto considerando

\footnotetext{
${ }^{1}$ Si se quisiera saber más sobre el proyecto, se podría revisar la página web: http://musicat.unam.mx/. En ella se encuentran publicaciones, documentos, así como información de eventos y algunos otros hallazgos de carácter académico.
} 
la distancia crítica que hay entre el lector y el tiempo en el que estos fueron producidos.

Tomando lo anterior en cuenta, en el Seminario de Música en la Nueva España y el México Independiente nos hemos centrado en formalizar un análisis crítico de los tipos de definiciones que se han hecho hasta el momento (previo a la publicación de este manuscrito) y se ha propuesto un modelo de definiciones terminológicas para el futuro glosario.

Por último, hay que decir que en este artículo se presenta una exposición razonada de cómo se llegó a la propuesta final de definiciones, cómo esta propuesta se puede aplicar a algunos ejemplos y, naturalmente, se ofrecen un breve contexto de la trayectoria del proyecto y un marco de referencia terminográfico y lexicográfico sobre los tipos de definiciones.

\section{2. "Musical" y un modelo de definiciones para su glosario}

El proyecto "MUSICAT" y su correspondiente Seminario de Música en la Nueva España y el México Independiente constituyen un trabajo de carácter multidisciplinario en el que se pretende anotar y formalizar el acceso sistemático a los documentos litúrgicos virreinales de México, mediante productos informáticos, de acceso abierto, y en línea. De forma aplicada, esta iniciativa de investigación pretende construir una herramienta de almacenamiento de datos y un motor de búsqueda que incluyera tópicos de investigación de disciplinas como la musicología, la historia, la historia del arte, la museografía y la restauración (Seminario de Música en la Nueva España y el México Independiente, 2009).

Entre múltiples logros, este proyecto ha conseguido publicar en línea dos bases de datos (la Base de Datos de Actas de Cabildo y otras ramas, y los Catálogos de música), una librería de cantorales (Libros de coro), así como una amplia selección de partituras (Colección Estrada) (Seminario de Música en la Nueva España y el México Independiente, 2009). Actualmente se ha incorporado a las líneas de investigación un trabajo enfocado a establecer una serie de descriptores que servirán como referentes temáticos en las búsquedas de los catálogos y bases de datos, y un glosario que servirá para clarificar asuntos, instrumentos, nombramientos, espacios, nombres y diferentes términos que no son de fácil acceso conceptual. En este trabajo únicamente se tratará el caso del glosario de términos.

También, en el Seminario de Música en la Nueva España y el México Independiente se reconoce la conveniencia de definir el léxico asociado a la práctica musical y litúrgica, esto para incrementar los conocimientos del usuario de las bases de datos. Algunos de estos términos, los de carácter litúrgico y musical, aparecen en las bases de datos resaltados en azul y, una vez que se posiciona el cursor del mouse sobre ellos, se abre un globo con una definición que se ha construido ad hoc. Asimismo, se resaltan arcaísmos ${ }^{2}$ relacionados con el habla de la época, los que serán definidos en el glosario propuesto (Seminario de Música en la Nueva España y el México Independiente, 2009) 3 .

\footnotetext{
2 Para efectos prácticos, se considerará la definición propuesta por Martínez de Sousa (2009), en la que se define a los arcaísmos como "formas léxicas o construcciones sintácticas que pertenecen a un estado de lengua desaparecido o en vías de desaparición, conservados en territorios en los que, pese a mantener cierta homogeneidad lingüística, se producen diferenciaciones dialectales".

${ }^{3}$ Se puede adelantar que el proceso de documentación de este léxico es un muestreo por conveniencia (Casal y Mateu, 2003) que supone esta metodología: si un especialista revisa un documento, previo a su publicación en las bases de datos, y se percata de una unidad léxica con cualquiera de las dos
} 
Grosso modo, la presente publicación responde a una necesidad de crear definiciones científicas de estos términos y a contar con un modelo específico para el proyecto. En los siguientes apartados se explica cómo, mediante un minucioso análisis lingüístico, se ha logrado construir un modelo definitorio para el glosario.

\section{Un marco teórico lingüístico para el proyecto}

Debido a que el proyecto de glosario propuesto desde el Seminario de Música en la Nueva España y el México Independiente tiene una inminente vocación lingüística y, más específicamente, cuenta con un enfoque terminográfico y de lexicografía especializada, en este apartado nos proponemos ofrecer un marco teórico suficiente que sostenga las decisiones aplicadas a la construcción del modelo definitorio. Para ello, a continuación se discute sobre los tipos de diccionarios, los componentes del artículo y las clases de definiciones que se reconocen.

\subsection{Los tipos de diccionarios}

Como es ampliamente sabido, disponemos de múltiples tipologías de obras de consulta; por señalar un ejemplo: Haensch y Lothar (1982), en el capítulo: “Tipología de las obras lexicográficas”, de La lexicografía. De la lingüística teórica a la lexicografía práctica, recopilan y explican, a lo largo de más de noventa cuartillas, un sinnúmero de criterios clasificatorios aplicables a los diccionarios existentes, no necesariamente excluyentes entre sí. Mencionan, por ejemplo, que los diccionarios se pueden clasificar por su enfoque (normativos o descriptivos), por sus características propias (formato o extensión, carácter lingüístico o enciclopédico, el número de lenguas, o la selección del léxico que sancionan), por su codificación (exhaustiva o selectiva), por criterios temporales (históricos o etimológicos), por su finalidad (onomásticos, paradigmáticos o de sinónimos, antónimos y parónimos), entre otros.

Sin embargo, pese a que existen tipologías tan amplias y diversas como las enunciadas por Haensch y Lothar, para efectos de esta investigación nos proponemos argumentar en favor de una tipología ad hoc basada en dos puntos de vista complementarios. En este sentido, una primera clasificación que consideramos es la de Lara Ramos (1996), en la que se argumenta en favor de la existencia de diccionarios lingüísticos, así como de diccionarios sociales.

En "Por una redefinición de la lexicografía hispánica", Lara Ramos (1996) explica que los diccionarios lingüísticos tienen un carácter puramente científico ${ }^{4} \mathrm{y}$ se enfocan en la caracterización del léxico, por ejemplo: los diccionarios de frecuencias, de ordenamiento inverso o de regionalismos, incluso. En palabras del autor, en un diccionario lingüístico "se plantea desde un principio y radicalmente el registro fidedigno de hechos léxicos tal como aparecen en un momento dado y en una comunidad lingüística determinada, como corresponde a todo programa de lingüística descriptiva de una lengua particular" (Lara Ramos, 1996, pp. 353-354).

En cambio, los diccionarios de carácter social refieren a obras de consulta que describen el significado de las palabras en un contexto social, incluso, desde un punto de vista normativo. En palabras del autor: "por ese carácter profundamente

características señaladas, la anota y, en trabajo colegiado, se consensua si se resaltará como término o arcaísmo.

${ }^{4}$ En este caso, "científico" remite a un enfoque de investigación descriptivista de la lengua; no debería confundirse con el sentido relativo al conocimiento de las ciencias humanas, en general. 
social de la normatividad y por su destino público prefiero llamarlos diccionarios sociales, que me parece una calificación más adecuada, por su amplitud y porque destaca su función social" (Lara Ramos, 1996, p. 354).

Por otro lado, Bergenholtz y Tarp (1995) aseguran que, desde un punto de vista funcional, se pueden reconocer diferencias entre la lengua para propósitos generales (LPG) y la lengua para propósitos específicos (LPE). Esta distinción supondría otra clase amplia de diccionarios, a saber: los diccionarios de LPE, los que, en cambio, ofrecen explicaciones o definiciones del concepto en sí y nunca ofrecerían información lingüística (Bergenholtz y Tarp, 1995), como en las clases descritas por Lara Ramos.

Asimismo, podemos decir que el propósito de un diccionario de LPE "is to describe and explain the way in which people who work in the relevant subject field structure their part of the world and their variety of language used to communicate about that field in order to help dictionary users to understand or use the terms in the way they are used in the subject field concerned" (Bergenholtz y Nielsen, 2013, p. 475$)^{5}$.

En este caso, y considerando los argumentos mostrados, se podría afirmar que la clasificación propuesta por Bergenholtz y Tarp (1995) supone la existencia de obras de consulta de LPG, independientemente de si se tiene un enfoque descriptivista del hecho léxico o social de este (Lara Ramos, 1996); y, por otra parte, reconoce la existencia de obras de consulta que sancionan LPE.

Adicionalmente, hay que decir que las obras de LPE también podrían tener dos orientaciones distintas: por un lado, las que tienen una vocación descriptiva, que simplemente constatan hechos específicos relativos al concepto; $y$, por otra parte, las obras prescriptivas, que fijan significados y norman sobre estos (Wüster, 1998). El panorama general de esta discusión puede verse de forma resumida en la ilustración 1.

Imagen 1. Una propuesta de tipos de diccionarios.

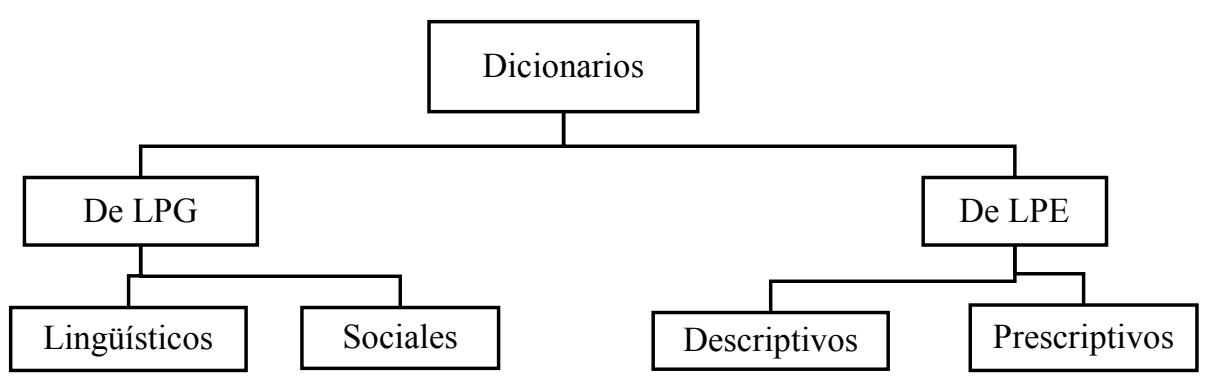

A la luz de esta propuesta clasificatoria, más adelante contrastaremos las diferencias existentes entre las clases: diccionarios de LPG y diccionarios de LPE, independientemente de su orientación; $\mathrm{y}$, más específicamente, discutiremos si esta diferenciación es relevante en términos de los tipos de definiciones de obras de consulta, objetivo central de esta investigación.

\footnotetext{
${ }^{5}$ Podemos agregar, sobre la conveniencia de esta clasificación, la idea de que las unidades léxicas suelen dividirse en palabras y términos, considerando que "[los términos] son al mismo tiempo iguales y diferentes a las unidades léxicas de una lengua, denominadas palabras en lexicología. Su carácter específico radica en sus aspectos pragmáticos y en su modo de significación. Su significado es el resultado de una negociación entre expertos que se produce dentro del discurso especializado [...]" (Cabré i Castellví, 1999).
} 


\subsection{El artículo, base de las obras de consulta}

El artículo lexicográfico es "la parte de un diccionario, glosario o vocabulario encabezada por una unidad léxica (el lema o entrada) y cuya finalidad es definirla o compararla" (Martínez de Sousa, 2009, p. 123). También conocido como artículo, este es considerado como base o fundamento del diccionario debido a que a partir de este se organiza y construye aquel (Porto Dapena, 2002).

El artículo está compuesto por tres elementos: la entrada, la ecuación sémica y la definición. La entrada constituye una unidad de recuperación, mención u ostensión del léxico o realidad citada en la obra; la ecuación sémica es un elemento, visible o no visible, que orienta la definición (este adquiere la forma de un verbo); y, por último, la definición refiere al contenido o significado de la entrada (Lara Ramos, 1997). Para explicar mejor esto, se puede ver la definición de "aguaardiente" del Diccionario de autoridades, Tomo I, que se reproduce a continuación:

Imagen 2. Artículo correspondiente a "aguaardiente" tomado del Diccionario de la Lengua Castellana.

Aguaardiente. s. f. Es la que por artificio se saca del vino, de sus heces, del trigo, y de otras cosas. Llámase assi este liquór, porque es claro como el agua, y porque arde echado fuego. Lat. Aqua ex vino vaporata. Aqua vinaria. El Sold. Pind. fol. 97. Pusieronme una cataplasma de estópas, y aguaardiente.

En la Ilustración 2, apelando a la conveniencia del ejemplo, se presenta el caso del artículo correspondiente a "aguaardiente", tomado del Diccionario de la lengua castellana (Real Academia Española, 1726), que permite ilustrar las tres partes del artículo. La primera, la entrada, se señala con un recuadro y contiene la palabra "aguaardiente" y las marcas "s. f."; luego, la ecuación sémica se resalta con el segundo recuadro y está representada con el verbo "ser", en este caso es visible; por último, la definición es el texto que se cita a continuación: "la que por artificio se saca del vino, de sus heces, del trigo, y de otras cosas. Llámase assi este liquór, porque es claro como el agua, y porque arde echado fuego. Lat. Aqua ex vino vaporata. Aqua vinaria. El Sold. Pind. fol. 97. Pusieronme una cataplasma de estópas, y aguaardiente" (Real Academia Española, 1726), misma que se subraya en la ilustración.

En este punto, resulta natural preguntarnos si existen diferencias sustanciales entre el artículo en un diccionario de LPG y uno de LPE. Ciertamente, a partir de revisar los dos tipos de diccionarios, podemos afirmar que, esencialmente, no hay diferencia alguna, ya que los artículos en los diccionarios de LPE también cuentan con entrada, ecuación sémica y definición, tal y como ocurre con los diccionarios de LPG. ${ }^{6}$ La ilustración 3 muestra un ejemplo que apoya esta afirmación.

\footnotetext{
${ }^{6}$ Aunque hemos señalado que los elementos constitutivos del artículo no se diferencian para el caso de un diccionario de LPG y uno de LPE, queremos aclarar que en el caso de la ecuación sémica sí hay algunos tipos de ecuaciones que se usan en obras de LPG, otros que se usan para obras de LPE y algunos que comparten ambas. Puesto que el objetivo de esta investigación no es discutir sobre las diferencias de la orientación de las ecuaciones sémicas, nos permitimos recomendar al lector un par de lecturas básicas en las que se hace referencia a esto: (1) Lara Ramos, L. F. (1997). Teoría del diccionario monolingüe. México, D.F.: El Colegio de México; y (2) Aguilar, C. A. (2008). Análisis
} 
Wado kaiho Una de las "doce monedas" dinásticas (jiuni sen*), emisiones* japonesas que imitan las piezas chinas tipo cash*. Fue acuñada por el Gobierno imperial en 708 .

La Ilustración 3 representa el caso del artículo correspondiente a "wako kaiho", tomado del Diccionario de numismática (Alfaro Asins, Marcos Alonso, Otero Morán y Grañeda Miñón, 2009). Como se puede observar, la entrada se encuentra en un recuadro y la definición está subrayada. En este caso, no se tiene una ecuación sémica visible ${ }^{7}$, pero se puede deducir que podría tratarse del verbo "ser" o, quizás, "designar".

No obstante podemos afirmar que no hay diferencias entre los artículos en las obras de LPG y las de LPE y corroborarlo con ejemplos como los de las ilustraciones 2 y 3 ; esta tesis también encuentra sustento en lo que ya hemos señalado antes (en la nota 5), a saber, las palabras y los términos son esencialmente iguales, salvo porque se diferencian en la forma en la que significan y designan cosas, respectivamente. Estas diferencias no son relevantes para la estructuración del artículo y para su diferenciación. Aunque, como se verá a continuación, para el caso de la definición de términos ${ }^{8}$ y palabras sí se podrían identificar algunas diferencias.

\subsection{Los tipos de definiciones}

Para Porto Dapena (2002) resulta pertinente hacer una distinción entre las definiciones de tipo conceptual y las definiciones explicativas. El autor explica que las de tipo conceptual expresan el contenido significativo del definido y que constituyen toda una clase que agrupa, a su vez, a otros tipos; mientras que las explicativas, también conocidas como definiciones por oposición (Rey-Debove, 1999), informan valores, funciones o usos de las palabras y, en particular, están asociadas a clases de unidades léxicas con significado relacional.

Esta distinción establece un límite claro entre las definiciones de obras que sancionan LPG y de las que recogen LPE. Esto es, las definiciones por oposición, explicativas o funcionales, que informan sobre cómo se relacionan ciertas unidades léxicas en el plano morfosintáctico, contextual y pragmático, son exclusivas de obras de LPG, ya que resulta muy inusual, prácticamente atípico, que un diccionario de LPE recoja y dé una explicación sobre clases de palabras como pronombres o adverbios.

Un ejemplo dado por Porto Dapena (2002) es la definición del pronombre su, que a continuación se cita: "Su, sus. Forma del pronombre posesivo de tercera persona en género masculino y femenino y en ambos números singular y plural que

lingüístico de definiciones en contextos definitorios. Universidad Nacional Autónoma de México, https://cesaraguilar.weebly.com/uploads/2/7/7/5/2775690/phdtesis first_version01.pdf.

${ }^{7}$ Para ahondar en el tema y comprender mejor la condición de que una ecuación sémica sea visible o no, se recomienda la lectura del capítulo: "El contenido proposicional del acto: la ecuación sémica" (pp. 131-166), del libro: Teoría del diccionario monolingüe (Lara Ramos, 1997). La referencia completa se encuentra en la bibliografía.

${ }^{8}$ Por si el que lo leyere lo considerara necesario, una definición operativa del concepto "término" sería esta: "unidad léxica de carácter técnico cuyo significado expresa un concepto propio de una lengua de especialidad” (Luna Traill, Vigueras Ávila y Baez Pinal, 2005, §1408). 
se utiliza antepuesto al nombre" (Porto Dapena, 2002: 282). Un caso como este nunca se encontraría en un diccionario de LPE, ya que no es uno de los propósitos de una obra de este tipo (Véase la definición de diccionario de LPE que se dio antes, en el §3.1).

Posiblemente, dentro de las definiciones conceptuales, una de las más comunes es la definición por inclusión que, según Rey-Debove (1999), es aquella en la que se asigna una clase general al definido $\mathrm{y}$, mediante el uso de un especificador, se distingue a este de otros elementos coordinados. Kipfer (2013) explica que estos elementos se llaman genus y differentia, respectivamente, y que el primero tiene la función de clasificar a la unidad léxica, funciona como el superordinado o hiperónimo directo del concepto; mientras que la differentia es un una unidad léxica o frase que caracteriza o cualifica al genus.

En la literatura relacionada con el tema existen diferentes nombres para este tipo de definición, a saber: definición de palabras llenas (Lara Ramos, 1994), definición por comprensión (Wüster, 1998), definición analítica (Aguilar, 2008; Kipfer, 2013), definición aristotélica (Baca Ibarra, Sierra Martínez, Aguilar y Barceló, 2006), definición por características circunstanciales (Dubuc, 1999), entre otras.

Este tipo de definiciones se usa tanto para diccionarios de LPG como para diccionarios de LPE. En la ilustración 4 se presenta un ejemplo prototípico de este tipo de definición; en él se resalta en un recuadro el genus, "Tinaja", y se subraya la differentia, "para conservación y transporte de las producciones medievales". El ejemplo fue tomado del Diccionario de materiales cerámicos (Padilla, Maicas y Cabrera, 2002).

Imagen 4. Definición correspondiente a "alfabía" tomada del Diccionario de materiales cerámicos.

Alfabia. Tinaja* para conservación y transporte de las producciones medievales.

Otra clase de definiciones que también funcionan para LPG y LPE es la constituida por las definiciones funcionales, las que se dan cuando "se hace explícita la diferencia específica, la cual ofrece una definición de un concepto a partir de su uso o aplicación en una situación dada" (Aguilar, 2008: pp. 51-52). Este tipo de definiciones introducen una explicación sobre la finalidad de un concepto, por ejemplo: "El relevador auxiliar [término] es usado para [predicación] asistir en el desarrollo de sus funciones a los relevadores de protección, como respaldo [definición]" (Aguilar, 2008, p. 56).

También queremos señalar que existen dos tipos de definiciones que, por su naturaleza, siempre se asocian con obras de LPE; estas son las definiciones por extensión y las definiciones enciclopédicas. Por un lado, las definiciones por extensión o extensionales constituyen "una enumeración de la extensión de un concepto, es decir, la enumeración de todos los conceptos específicos que se encuentran al mismo nivel dentro del sistema de conceptos". Por otro lado, la definición enciclopédica es una "explanation of a word or phrase that is comprehensive and includes information that is not strictly linguistic, a definition that reflects encyclopedic knowledge facts" (Kipfer, 2013, p. 395).

A continuación se citan dos ejemplos de estos tipos: la definición extensional se ilustra a partir de un contexto definitorio, citado por Aguilar (2008, p. 56): "La 
Terminal de contenedores [término] cuenta con [predicación] dos muelles de atraque, el muelle del Bufadero y el muelle del Dique del Este [definición]". Mientras que la definición enciclopédica se toma de la Wikipedia, del artículo correspondiente a Persia o la nación persa: “[...]Antes del surgimiento de la nación persa, la zona del Medio Oriente venía siendo azotada por las guerras. El foco de estas guerras era el pueblo agresor y militarista de Asiria. Los asirios constantemente lanzaban campañas contra los pueblos que los rodeaban, saqueando, efectuando matanzas y deportando a las poblaciones o a sus clases dirigentes por lo menos[...]" (Wikimedia Commons, 2019).

En general, se entiende que la definición es o funge como sinónimo de su definido (Porto Dapena, 2002). Esta es la razón por la cual se asume que un tipo común de definición es la sinonímica, o definición por sinónimos, en la que se establece una equivalencia conceptual con otra unidad léxica; estas definiciones se usan tanto en obras de LPG como en obras de LPE (Aguilar, 2008; Dubuc, 1999; Porto Dapena, 2002).

Existen otro tipo de definiciones mucho menos frecuentes, por ejemplo: la ostensiva o sintética, que incluye una representación gráfica del objeto o que, en su defecto, mediante el uso de una frase que hace una analogía con objetos o ideas comparables al concepto descrito (Kipfer, 2013); las intencionales, las que usan una fórmula para indicar que el concepto es parte de una clase superior (Wüster, 1998), Kipfer menciona que es muy común el uso de la fórmula "Kind of ...., en el inglés (Kipfer, 2013, p. 398); definiciones formulaicas, que recurren a una fórmula para construir la explicación (Kipfer, 2013), y combinadas, Wüster (1998, pp. 66-67) señala que puede haber combinaciones de tipos definitorios, extensión y comprensión.

Tabla 1. Uso reportado en diccionarios de los tipos de definiciones revisadas.

\begin{tabular}{|c|c|c|}
\hline Definiciones en obras de LPG & Definiciones en obras de LPG y LPE & Definiciones en obras de LPE \\
\hline $\begin{array}{l}\text { Definición por oposición o } \\
\text { definición explicativa. }\end{array}$ & $\begin{array}{l}\text { - Definición analítica, definición aristotélica, } \\
\text { definición por inclusión, definición por } \\
\text { comprensión, definición de palabras llenas } \\
\text { o definición por características. } \\
\text { - Definición funcional. } \\
\text { - Definición sinonímica o definición por } \\
\text { sinónimos. } \\
\text { - Definición ostensiva o sintética. } \\
\text { - Definición formulaica. }\end{array}$ & $\begin{array}{l}\text { - Definición por extensión o } \\
\text { extensional. } \\
\text { - Definición enciclopédica. } \\
\text { - Definición intencional. } \\
\text { - Definicón combinada. }\end{array}$ \\
\hline
\end{tabular}

Como se puede ver en la tabla 1, 4 de 10 clases de definiciones reconocidas son exclusivas de diccionarios de LPE (definiciones extensionales, enciclopédicas, intencionales y combinadas), 5 de 10, son modelos compartidos entre diccionarios de LPG y LPE (definiciones analíticas, funcionales, sinonímicas, ostensivas y formulaicas); mientras solo un tipo es exclusivo de obras de consulta de LPG (definición por oposición).

En el contexto de lo que pretendemos explicar, nos quedaremos con 9 de los 10 tipos señalados para nuestro análisis y pondremos particular énfasis en una clase de definiciones, las combinadas explicadas por Wüster (1998), ya que el enfoque principal de este proyecto corresponde a un glorario de LPE. En el capítulo siguiente se verá el porqué de esta decisión. 


\section{Diseño de un corpus de definiciones}

Para delimitar cuál sería el tipo o tipos de definiciones más comunes hechas en el proyecto y, con base en esto, crear una propuesta definitoria ad hoc, se construyó un corpus, se anotó y se analizaron cuantitativamente sus datos. A continuación se ilustra cuáles son las variables del corpus y sus atributos.

Después de haber explicado los tipos de definiciones que podríamos adoptar, en este apartado nos proponemos explicar cómo organizamos y anotamos un corpus de definiciones, con miras a evaluar cuál es el tipo de definición que más conviene para nuestro glosario.

La idea de contar con un pequeño corpus de esta naturaleza se justifica si se considera que ello nos permitiría tomar una mejor decisión sobre el estilo de explicación que un conjunto de especialistas brinda sobre la terminología que utiliza. Esto es, por lo menos ocho especialistas, en trabajo colegiado, definieron más de 165 términos y arcaísmos que aparecen en documentos especializados de la música litúrgica.

En nuestra opinión, considerar estas definiciones, que se hicieron sin ninguna experiencia o a partir de algún conocimiento de los modelos definitorios revisados, podría ser una referencia para anticipar el uso de un tipo de definiciones relativamente frecuentes para contextos de LPE y observar si existe algún condicionamiento relacionado con el tipo de unidad léxica o dominio al que pertenecen estas unidades.

Ya se ha explicado que durante las reuniones periódicas del seminario se identifican términos y arcaísmos léxicos que, en trabajo colegiado, se han ido definiendo. El proyecto, justo antes de la publicación de este trabajo, contaba con un registro de 165 unidades léxicas y sus correspondientes definiciones, todas ellas elaboradas por especialistas vinculados al proyecto, formados en disciplinas como la musicología, la historia, la historia del arte, la museografía y la restauración. Esas 165 definiciones constituyen la base de análisis de esta investigación.

Las definiciones fueron organizadas en una tabla de Excel siguiendo estos criterios: primero, asignamos a cada uno de los términos un identificador numérico único e irrepetible; estos identificadores se sancionan en la primera columna del tablón (columna A) y funcionan como un control interno del léxico; segundo, los términos y arcaísmos se incluyen en la segunda columna (columna B); y tercero, las definiciones propuestas hasta el momento se incluyen en la columna $\mathrm{C}$ del tablón de Excel.

Para este último caso, el de la columna $\mathrm{C}$, nos dimos cuenta de que en la captura de los datos podría haber algunas formas homónimas agrupadas en una misma casilla en la que se definirían varios conceptos inscritos bajo una sola forma léxica. Esto sería equivalente a incluir en el mismo artículo varios conceptos que no tienen relación semántica; un caso es el de "manual", que son tres formas homónimas que designan tanto 'a una compensación económica por prestar servicios en la iglesia', así como 'un tipo de libro relacionado con el ritual catedralicio' y 'un órgano de funcionamiento manual'. Por ello, separamos las formas homónimas siguiendo este criterio: cada registro deberá tener un identificador, un término y una sola definición; en el caso de que una forma léxica esté relacionada con dos o más conceptos, se tendrán que crear varios registros. Con esa adecuación, la base de datos creció hasta contar con 167 registros; en ese momento, la base de datos se veía así: 
Tabla 2. Uso reportado en diccionarios de los tipos de definiciones revisadas.

\begin{tabular}{|l|l|l|}
\hline A & B & C \\
\hline ID & Término & Definición Musicat \\
\hline $\mathbf{1}$ & acólito & $\begin{array}{l}\text { Ministro de la iglesia que ha recibido las cuatro órdenes menores del orden sacerdotal (ostiario, } \\
\text { lector, exorcista y acólito). Su obligación es servir al altar. }\end{array}$ \\
\hline $\mathbf{2}$ & aderezo & Adorno o compostura. \\
\hline $\mathbf{3}$ & adviento & Ciclo o tiempo inicial del año litúrgico. \\
\hline $\mathbf{4}$ & agonías & Toque de campanas para pedir por la buena muerte de los agonizantes. \\
\hline
\end{tabular}

Para la descripción lingüística de los tipos de definiciones agregamos al tablón, en las columnas $\mathrm{D}$ a la $\mathrm{H}$, los siguientes campos de información: en la columna D se aclara si el ítem léxico definido correspondía a un término o a un arcaísmo; la columna E, llamada "extensión", describe si la definición es simple o combinada; en la columna $\mathrm{F}$ se explica el tipo de definición, sea extensional, enciclopédica, intencional, analítica, funcional, sinonímica, ostensiva o formulaica; $\mathrm{y}$, por último, en la columna $\mathrm{G}$ se describe el dominio al que pertenece el ítem léxico (artefactos, asuntos laborales, cargos y oficios, géneros musicales, liturgia, indumentaria, instrumentos musicales, léxico general, liturgia y misceláneos) ${ }^{9}$. La base de datos se ve así:

Tabla 3. Vista del tablón de Excel completo.

\begin{tabular}{|l|l|l|l|l|l|l|}
\hline A & B & C & D & E & F & G \\
\hline ID & Término & Definición Musicat & Sentido & Extensión & Tipo de definición & Dominio \\
\hline $\mathbf{1}$ & acólito & $\begin{array}{l}\text { Ministro de la iglesia que ha } \\
\text { recibido las cuatro órdenes } \\
\text { menores del orden sacerdotal } \\
\text { (ostiario, lector, exorcista y } \\
\text { acólito). Si obligación es servir } \\
\text { al altar. }\end{array}$ & Término & Combinada & Analítica / funcional & $\begin{array}{l}\text { Cargos y } \\
\text { oficios }\end{array}$ \\
\hline $\mathbf{2}$ & aderezo & Adorno o compostura & Arcaísmo & Simple & Sinonímica & Léxico general \\
\hline $\mathbf{3}$ & adviento & $\begin{array}{l}\text { Ciclo o tiempo inicial del año } \\
\text { litúrgico. }\end{array}$ & Término & Simple & Analítico & Liturgia \\
\hline $\mathbf{4}$ & agonías & $\begin{array}{l}\text { Toque de campanas para pedir } \\
\text { por la buena muerte de los } \\
\text { agonizantes. }\end{array}$ & Término & Simple & Analítica & Liturgia \\
\hline
\end{tabular}

A partir de la estructura de datos de este tablón se hacen algunas inferencias que nos permitieron formalizar la propuesta de definición. En el siguiente apartado ofrecemos una interpretación de los datos analizados.

\section{Aproximación cuantitativa a las variables analizadas}

El primer paso del análisis cuantitativo de los datos del corpus de definiciones implica mostrar una aproximación general a las columnas que planteamos como variables de análisis ("sentido" o si la unidad léxica es un término o arcaísmo; "tipo de definición", descrita a partir de las clases que se han revisado antes; extensión o si la definición es simple o combinada; y el dominio o las temáticas planteadas para clasificar el léxico).

\footnotetext{
${ }^{9}$ Estos dominios se han consensuado en el seminario y describen cómo se ha organizado el léxico del área del saber, desde el enfoque del propio proyecto. Aclaramos que esta clasificación es dinámica y que, mientras avanza el proyecto, se han ido agregando clases a esta organización conceptual. No se descarta que más adelante se agreguen otras clases que deban considerarse.
} 
Las tendencias generales que arrojan nuestros datos se pueden resumir así: en general, el corpus recoge 144 términos (86.2\% del total) y 23 arcaísmos (13.8\%); considerando la extensión de las definiciones, desde un punto de vista general, se tienen 127 definiciones simples que representan el $76 \%$ del total de registros y 40 definiciones combinadas que representan el restante $24 \%$.

Los tipos de definiciones que se documentan son del tipo analítica, enciclopédica, extensional, funcional, intensional y sinonímica, únicamente; y, como era esperado, todos los dominios se encuentran representados, ya que son el resultado de una clasificación definida a priori, aunque, debido a que la muestra de definiciones es tan pequeña y no todos los dominios se relacionan con todas las variables anteriores, este análisis se presenta sin una relación con las variables anteriores. A continuación se muestra la forma en la que el resto de las variables interactúan, desde la perspectiva del tipo de unidad léxica que se define, a saber: si es un arcaísmo o un término, respecto al tipo de definición y su extensión.

Los arcaísmos (23 casos) son explicados mediante definiciones simples y combinadas, en esta proporción: las definiciones simples son 17, es decir, 73.9\% del total de definiciones de arcaísmos, mientras que las definiciones combinadas son 6 o el $26.1 \%$.

Las definiciones simples se formalizan a partir de dos tipos: definiciones analíticas y sinonímicas. De las definiciones analíticas se tienen 11 casos y de las definiciones sinonímicas solo 6. En (1) y (2) se presentan un par de ejemplos de ellas, la primera analítica y la segunda sinonímica.

1) 59 cuadrante. Folio cuadriculado en el que se llevaba el control de las asistencias, multas y salarios de los miembros del coro catedralicio.

2) 67 empavesar. Engalanar o arreglar.

Por otra parte, es notable que las definiciones combinadas están construidas a partir de, primero, una definición analítica que es complementada por una definición funcional y una sinonímica (un único caso); y, segundo, con una definición analítica concatenada a otra definición analítica (cinco casos). Posiblemente esto revele que los especialistas recurren a estas combinaciones para enriquecer la explicación sobre el léxico y, desde nuestro punto de vista, es un indicio de la forma en la que ellos consideran que resulta más claro presentar una explicación del léxico mediante una definición. Las combinaciones de estas definiciones y un ejemplo de ellas se presentan en la siguiente tabla.

Tabla 4. Tipos de definiciones combinadas para los arcaísmos.

\begin{tabular}{|l|l|}
\hline Combinación de definiciones & Artículo \\
\hline Analítica/Analítica & $\begin{array}{l}147 \text { sacristán. Ayudante de una iglesia encargado de cuidar y preparar los } \\
\text { ornamentos y objetos sagrados de la misma. Dignidad eclesiástica a cuyo } \\
\text { cargo está el cuidado de los objetos sagrados de una iglesia. }\end{array}$ \\
\hline Analítica/Funcional/Sinonímica & $\begin{array}{l}135 \text { pertiguero. Ministro o ayudante laico (no clérigo) que asiste acompañando } \\
\text { a los ministros de la liturgia en las celebraciones catedralicias revestido con su } \\
\text { atuendo particular, llevando una pértiga en la mano. Su función específica era } \\
\text { la de echar fuera del recinto eclesial a los animales callejeros durante las } \\
\text { celebraciones. Por eso también se le conocía como perrero. }\end{array}$ \\
\hline
\end{tabular}

El ejemplo de definición Analítica/Analítica que se presenta en la tabla anterior es un caso muy interesante que representa a todas las definiciones semejantes. Como se puede adelantar, la idea de plantear dos definiciones analíticas 
concatenadas es impráctica, ya que estas se podrían refundir en una sola, por ejemplo, nosotros recomendaríamos que sacristán se presente con su definición como en el ejemplo (3).

3) 147 sacristán. Dignidad eclesiástica encargada de cuidar y preparar los ornamentos y objetos sagrados de la misma.

De forma práctica, si refundiéramos las cinco definiciones del tipo Analítica/Analítica siguiendo la propuesta presentada en (3), cosa que resulta a todas luces funcional, podríamos reagrupar los tipos de definiciones asociadas con arcaísmos de esta forma, para dar un panorama global.

Imagen 5. Tipos de definiciones para los arcaísmos, panorama general.

Definiciones combinadas (analíticas/funcionales/sinonímicas); 1

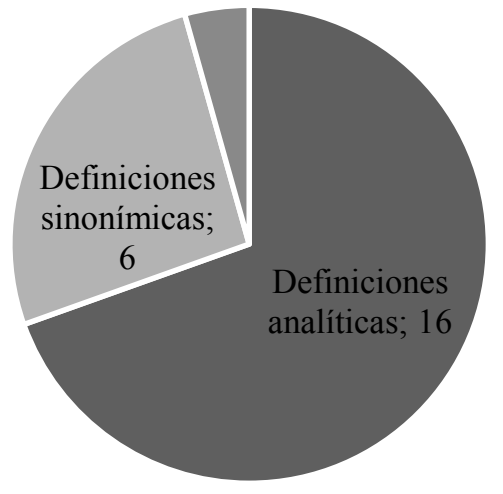

En esta ilustración se presentan todos los tipos de definiciones de arcaísmos agrupados, siempre y cuando se considere que las definiciones analíticas/analíticas se refundieran en una sola. Este ejercicio se plantea para simplificar el análisis propuesto y describir que las definiciones analíticas son comúnmente usadas para definir los arcaísmos del proyecto; después, las definiciones sinonímicas son un recurso menos común, pero usado para ello; y, por último, se recurre al uso de una definición combinada que se construye a partir de una analítica, una sinonímica y una funcional.

Debido a que registramos pocos arcaísmos, no se puede formalizar un análisis del dominio respecto a los tipos de definiciones, pero sí se describe que los arcaísmos se relacionan temáticamente con cinco de nueve dominios, a saber: asuntos laborales, cargos y oficios, indumentaria, liturgia y misceláneos. La distribución de los dominios respecto a los arcaísmos es la siguiente:

Tabla 5. Distribución de los arcaísmos por dominio.

\begin{tabular}{|l|l|}
\hline Dominio & Frecuencia, \% \\
\hline asuntos laborales & 5 de $23,22 \%$ \\
\hline cargos y oficios & 2 de $23,8,6 \%$ \\
\hline indumentaria & 3 de $23,13 \%$ \\
\hline liturgia & 2 de $23,8,6 \%$ \\
\hline misceláneos & 11 de $23,47,8 \%$ \\
\hline
\end{tabular}


Como se puede ver en la tabla anterior, los arcaísmos tienden a ser conceptos generales o a asociarse con el dominio miscelánea. Esto es absolutamente esperado, ya que estos arcaísmos, en su momento, fueron LPG vitales y no se organizan dentro de los dominios relativos a la música litúrgica.

Los términos, por otra parte, en la base de datos de definiciones, son el $86.2 \%$ del total de registros o, lo que es lo mismo, el corpus cuenta con 144 términos y sus respectivas definiciones. De estos 144 registros, las definiciones simples son 110, es decir, $76.4 \%$ del total de definiciones de términos, mientras que las combinadas son el $23.6 \%$ (34 casos).

Si comparamos estas cifras con las presentadas en el apartado anterior respecto al mismo punto, veríamos que no existe una diferencia significativa entre la extensión de definiciones para arcaísmo y la extensión para términos, ya que se podría decir que 7 de cada 10 definiciones de arcaísmos son simples; mientras que para los términos tenemos que 8 de cada 10 también son definiciones simples, diferencia que no sería significativa. Los porcentajes de distribución para la comparación de estos dos casos se ilustran en la tabla siguiente.

Tabla 6. Extensión de las definiciones según el tipo de unidad léxica (presentado porcentualmente).

\begin{tabular}{|l|l|l|}
\hline & Para arcaísmos (\%) & Para términos (\%) \\
\hline Definiciones simples & 17 casos, 73,9\% (cerca de 7 de cada 10) & 110 casos, 76,4\% (cerca de 8 de cada 10) \\
\hline $\begin{array}{l}\text { Definiciones } \\
\text { combinadas }\end{array}$ & 6 casos, 26,1\% (cerca de 3 de cada 10$)$ & 34 casos, 23,6\% (cerca de 2 de cada 10) \\
\hline
\end{tabular}

Los tipos de definiciones simples que se usan para dar una explicación de los términos son analíticas, enciclopédicas, funcionales, intencionales y sinonímicas, es decir, existe una diferencia entre los arcaísmos y los términos que radica en que los segundos, además de los tipos coincidentes para los primeros, se explican con definiciones enciclopédicas, funcionales e intencionales (situación que corrobora lo explicado en apartados anteriores y que se resumió en la Tabla 1. Uso reportado en diccionarios de los tipos de definiciones revisadas). En (4), (5), (6), (7) y (8) se presentan ejemplos de ellas: la primera es una definición analítica, y, después, le siguen una enciclopédica, una funcional, una intencional y una sinonímica, en ese orden.

4) 19 asistente de coro. Servidor que canta en el coro del Oficio Divino y en otras ocasiones como los aniversarios.

5) 109 magnificat. El magníficat solía ser interpretado antes de las misas de festividades religiosas extraordinarias. Adquirió su nombre debido a que "magnificat" era la primera palabra del Canto de la Virgen María (Lucas, 1, 46-55) con que culmina el oficio de vísperas. Este carácter introductorio le confiere una extensión menor a la de las cantatas.

6) 14 apuntador. Encargado de llevar el registro de retardos o faltas de los miembros del coro catedralicio, así como las irregularidades en su comportamiento.

7) 7 alborada. Un tipo de toque de campanas.

8) 115 ministril. Músico. 
El porcentaje de estos tipos de definiciones se representa en la tabla siguiente $\mathrm{y}$, adicionalmente, se agrupan los tipos menos comunes con miras a dar una interpretación complementaria (esto se presenta inmediatamente después de la tabla):

Tabla 7. Tipos de definiciones simples para el caso de los términos (presentados porcentualmente).

\begin{tabular}{|c|c|c|c|c|c|}
\hline & $\begin{array}{l}\text { Definiciones } \\
\text { analíticas }\end{array}$ & $\begin{array}{l}\text { Definiciones } \\
\text { sinonímicas }\end{array}$ & $\begin{array}{l}\text { Definiciones } \\
\text { enciclopédicas }\end{array}$ & $\begin{array}{l}\text { Definiciones } \\
\text { funcionales }\end{array}$ & $\begin{array}{l}\text { Definiciones } \\
\text { intencionales }\end{array}$ \\
\hline Porcentaje individual & 92 casos, $83,6 \%$ & 9 casos, $8,2 \%$ & 5 casos, $4,6 \%$ & 3 casos, $2,7 \%$ & 1 caso, $0,9 \%$ \\
\hline $\begin{array}{l}\text { Porcentaje reagrupando los casos } \\
\text { menos frecuentes }\end{array}$ & 92 casos, $83,6 \%$ & \multicolumn{4}{|c|}{18 casos, $16,4 \%$} \\
\hline
\end{tabular}

De la información presentada en la tabla 7 se puede obtener una conclusión preliminar: la definición analítica, para el caso de las definiciones simples de términos, es la más común para definirlos, aunque aún faltaría analizar el panorama para las definiciones compuestas. El tipo analítico representa casi 8 de cada 10 de los casos analizados, mientras que las definiciones enciclopédicas, funcionales, intencionales y sinonímicas representan, en suma, cerca de 2 de cada 10 en este análisis particular (esto se corrobora en la reagrupación presentada en la última fila).

Las definiciones combinadas que explican el significado de los términos son el 23.6\% (34 casos), mucho menos que las simples (cerca de 2 de cada 10 definiciones registradas). Estos tipos se componen a partir de dos clases de definiciones principales: analíticas y sinonímicas, esto es, la primera definición es una analítica o una sinonímica y, después, se complementan mediante otros tipos. El porcentaje de estas se presenta en la tabla siguiente; además, presentamos una lista adicional, en la columna de la derecha, de los tipos que les siguen.

Tabla 8. Definiciones combinadas y su complemento con otras definiciones (presentado porcentualmente).

\begin{tabular}{|l|l|l|}
\hline Definición principal & Frecuencia $(\%)$ & Tipos que la complementan \\
\hline Analítica & $17,6 \%, 6$ casos & $\bullet$ Analítica \\
& & $\bullet$ Enciclopédica \\
& & $\bullet$ Extensional \\
& & $\bullet$ Funcional \\
& & $\bullet$ Sinonímica \\
\hline Sinonímica & $82,4 \%, 28$ casos & $\bullet$ Enciclopédica \\
\hline
\end{tabular}

Las definiciones analíticas con sus complementos se presentan en los ejemplos (9) al (13); estos corresponden a definiciones analíticas en su combinación con otras analíticas, enciclopédicas, extensionales, funcionales y sinonímicas. Los ejemplos se presentan en ese orden.

9) 121 motete. Forma musical exclusiva de la polifonía renacentista. Composición musical para varias voces humanas de distinta tesitura sobre un texto litúrgico en latín.

10) 34 cantor. Miembro de un coro o grupo de música vocal. Antiguamente, esta palabra también indicaba una jerarquía dentro de la organización musical de una iglesia como autoridad responsable de la presencia de la música en los servicios litúrgicos. 
11) 28 breviario. Libro que contiene los textos del oficio de alabanza cotidiana a Dios. Generalmente consta de cuatro tomos correspondientes a las estaciones del año: Adviento y Navidad, Cuaresma y Pascua y dos libros más del tiempo ordinario.

12) 26 bayeta. Tela de lana, floja y poco tupida. Sirve para ornamentar, forrar o cubrir objetos en el ritual catedralicio.

13) 8 almucias. Vestimenta eclesiástica que confiere dignidad. Muceta.

Igual que ocurre en el caso de las definiciones analíticas complementadas por otra analítica, que se explicaron para el caso de los arcaísmos, creemos que en un ejemplo como el de (9) se podrían refundir las definiciones, una propuesta sería la presentada en (14):

14) 121 motete. Composición musical renacentista sobre un texto litúrgico en latín, exclusiva de la polifonía, elaborada para varias voces humanas de distinta tesitura.

Por otro lado, cuando las definiciones sinonímicas fungen como principales, siempre van acompañadas de una definición enciclopédica, un ejemplo de esto se presenta en (15).

15) 41 chanzoneta. Cancioncilla. Este término se usó como denominación genérica de los villancicos o cualquier otra composición en lengua distinta al latín: español, portugués, gallego, náhuatl, etc. de las que se usaban en las celebraciones litúrgicas de determinadas fiestas.

Tabla 9. Distribución de los términos por dominio

\begin{tabular}{|l|l|}
\hline Dominio & Frecuencia, \% \\
\hline artefactos & 16 de $144,11,1 \%$ \\
\hline asuntos laborales & 8 de $144,5,6 \%$ \\
\hline cargos y oficios & 34 de $144,23,6 \%$ \\
\hline difuntos & 4 de $144,2,8 \%$ \\
\hline géneros musicales & 4 de $144,2,8 \%$ \\
\hline indumentaria & 6 de $144,4,2 \%$ \\
\hline intrumentos musicales & 11 de $144,7,6 \%$ \\
\hline liturgia & 31 de $144,21,5 \%$ \\
\hline misceláneos & 30 de $144,20,8 \%$ \\
\hline
\end{tabular}

Como se puede adelantar, existen diferencias entre los dominios con los que se relacionan los términos y los arcaísmos. Para el caso de los términos, los dominios que los recogen se relacionan con los artefactos, asuntos generales, difuntos y géneros musicales; suponemos que esto tiene que ver con que se relacionan con la terminología de la música litúrgica, directamente. Sin embargo, como ya se ha dicho antes, en este momento no contamos con una amplia terminología, además de que la categoría misceláneos se va a ir modificando $\mathrm{y}$, a partir de ella, se crearan otras categorías, por lo tanto, aventurar alguna explicación más profunda al respecto no tendría fundamento.

\section{El modelo de definición propuesto}

Considerando los datos analizados y la forma en la que se ha presentado el análisis del apartado anterior, la conclusión general sería: para el contexto de la música litúrgica y, en particular, del proyecto, una definición analítica bastaría para elaborar nuestras definiciones, sean para arcaísmos léxicos o para términos. Esto, en virtud 
de que resultan muy frecuentes, simplificaría el trabajo definitorio y, como se explicó antes, es un modelo útil tanto para LPG como de LPE.

Lo cierto es que encontramos ventajas en proponer un modelo de definición combinada que integre a los tipos usados por los especialistas del área, por ejemplo: una definición funcional complementa el alcance de la definición de artefactos, cargos y oficios; una extensional enuncia los elementos que componen a artefactos, indumentaria e instrumentos; y debido a que el proyecto del seminario se inscribe en un contexto de profesionales historiadores e historiadores del arte, no podíamos renunciar a una enciclopédica, ya que, en palabras de los colegas del seminario: "se perdería información valiosa para el contexto de la disciplina". En cuanto a las definiciones sinonímicas, es muy común que existan sinónimos del léxico con el que se trabaja y, por ello, no quisiéramos renunciar a dar cuenta de estas relaciones léxico-semánticas. Por último, decidimos no recurrir a definiciones intencionales, ya que solo tenemos un caso y lo hemos tratado como un hapax legomenon.

Es por ello, y a la luz de estas conclusiones, que desde el seminario consensuamos un modelo de definición dividida en dos partes: la primera, que llamamos definición corta, y que es aplicable a arcaísmo y términos; y, la segunda, que es de uso exclusivo para términos y que hemos llamado definición larga. Este modelo se explica y compara en la tabla 9.

Tabla 10. Modelos de definiciones propuestas

\begin{tabular}{|c|c|}
\hline Modelo de definición & Forma que adquiere \\
\hline Definición corta (arcaísmos) & - Definicón analítica (obligatoria) \\
\hline Definición larga (términos) & $\begin{array}{l}\text { - } \quad \text { Definición analítica (obligatoria) } \\
\text { Complementada por una: } \\
\text { - } \quad \text { Definición funcional } \\
\text { - } \quad \text { Definición extensional } \\
\text { - } \quad \text { Definición enciclopédica } \\
\text { - } \quad \text { Y una definición sinonímica (estas cuatro son } \\
\text { optativas y se aplican a la redacción según cada } \\
\text { caso). }\end{array}$ \\
\hline
\end{tabular}

Un ejemplo resultante de la aplicación de este modelo es el caso de "chantre", mismo que se transcribe a continuación, y del cual se comparan los cambios que se efectuaron a partir del modelo definitorio propuesto ${ }^{10}$. El caso de "chantre", originalmente, se definía así:

16) 40 chantre. Autoridad del cabildo, con rango de dignidad, a cuyo cargo estaba todo lo relacionado con el canto del ritual catedralicio.

Después de la revisión de la definición, a partir del modelo propuesto, el artículo se ve así:

17) 40 chantre. Dignidad o autoridad del cabildo encargada de todo lo relacionado con el canto en el ritual catedralicio. Entre sus funciones estaba mediar entre las autoridades del cabildo y los músicos, escribir o hacer escribir la tabla o matrícula de cada semana, advertir a otras autoridades lo que se iba a rezar, lo que se iba a cantar y atender las funciones administrativas del coro. En el Virreinato de la

\footnotetext{
${ }^{10} \mathrm{Al}$ final del documento, en el Anexo 1, se presenta una propuesta de guía rápida para la elaboración de definiciones creada para que resulte más sencilla la redacción de estas. Dicha guía refleja los resultados de la discusión que se ha planteado hasta ahora.
} 
Nueva España, los chantres podrían llevar a cabo actividades de compra de instrumentos, cuidar el aderezo de los órganos y la contratación de los músicos.

El contraste entre los ejemplos (16) y (17) revela que el modelo propuesto podría implicar una simplificación de la definición analítica de (17), en la que se mantiene la esencia de la definición original (16). Asimismo, la inserción de una definición funcional amplía el contenido de la definición enumerando todas las funciones asociadas a este cargo. Al final, la definición enciclopédica señala una excepción que se daba en el Virreinato, en casos poco comunes, en el territorio que hoy se conoce como México.

En suma, estos dos tipos de definiciones enriquecen la explicación del concepto $\mathrm{y}$, en nuestra opinión, generan un contexto más rico para que quien consulte las bases de datos entienda mejor el documento.

Por último, asumiendo que la definición elaborada pudiera dar cuenta parcialmente del significado de la noción definida, se propone un último paso para analizar y corroborar que esta es efectiva, esto es: se propone que el redactor, al momento de presentar la definición al resto de colegas, en las reuniones colegiadas, convierta la definición en una pregunta y la presente al resto investigadores.

Esto se ejemplifica retomando el ejemplo (17), que ya se ha referido antes, reformulando la definición de esta manera: ¿Cuál es la "Dignidad o autoridad del cabildo encargada de todo lo relacionado con el canto en el ritual catedralicio"? [que] Entre sus funciones estaba mediar entre las autoridades del cabildo y los músicos, escribir o hacer escribir la tabla o matrícula de cada semana, advertir a otras autoridades lo que se iba a rezar, lo que se iba a cantar y atender las funciones administrativas del coro. ¿En el Virreinato de la Nueva España, los chantres podrían llevar a cabo actividades de compra de instrumentos, cuidar el aderezo de los órganos y la contratación de los músicos?

$\mathrm{Si}$, en todos los casos, la respuesta a esa definición en forma de pregunta fuese el término definido, la definición será aceptada, en caso contrario, se harán las modificaciones pertinentes a la definición en trabajo colegiado.

\section{Resumen y conclusiones}

En el presente trabajo de investigación se ha puesto un acento especial sobre el hecho de reconocer las necesidades de una comunidad de práctica y, desde la teoría de la terminografía, ofrecer una solución aplicada y ad hoc para redactores que colaboren en el proyecto MUSICAT.

La idea de ofrecer un modelo útil para redactores de definiciones de términos de un área del saber no es un enfoque novedoso, ya que en la literatura se podrían encontrar trabajos como los de García de Quesada y Montero Martínez (2003), Valero Doménech (2007), Valero Doménech \& Alcina Caudet (2015), Azevedo Delvizio (2018), entre otros. Sin embargo, lo que hace diferente al presente trabajo, respecto a los anteriormente citados, es que nosotros hemos considerado como base empírica documentación primaria, es decir, las definiciones que los especialistas han creado, mientras que en los trabajos citados se consideran fuentes secundarias y terciarias, así como marcos teóricos.

A partir de asumir que hay diferencias entre la LPG y la LPE, y la revisión de la literatura tocante al tema, se pudo establecer que hay tipos de definiciones exclusivas para las obras de LPG, para diccionarios de LPE, y que existen tipos comunes a ambas. La revisión de los tipos de definiciones mostró que existen, 
cuando menos, diez clases distintas documentadas en la literatura; y aunque no todos se registraron en el estudio de caso, nos parece que es conveniente mencionarlos, por dos razones: primero, dar cuenta del estado de la cuestión; y, segundo, ya que no descartamos la idea de ampliar el corpus en una etapa futura de la investigación y, por tanto, documentar otros tipos de definiciones.

También se descarta la idea de que el artículo tendría una estructura diferenciada para obras de consulta de LPG y LPE.

Los datos analizados indican que la definición analítica es muy común, incluso, en casos de redactores que las usan y que desconocían totalmente del tema. Sin embargo, la decisión fue tomar como base la definición analítica y complementarla con definiciones funcionales, extensionales, enciclopédicas y sinonímicas, esto basado en la posibilidad de proponer definiciones combinadas (Wüster, 1998) y atendiendo las necesidades de los miembros de la propia comunidad.

Considerando que el perfil profesiográfico de los redactores de las definiciones es heterogéneo, tal y como se mencionó en el apartado 2, y asumiendo que todos los integrantes del proyecto serán redactores, se ha propuesto una "Guía rápida para la elaboración de definiciones”, disponible en el Anexo. 1. En esta guía se exponen los criterios que se deben considerar para la redacción de una definición, a saber: respondiendo preguntas específicas y completando frases hechas. Esta guía, pues, funge como una herramienta de acompañamiento para cada uno de los redactores.

Por último, sentimos que una línea de trabajo futura sería la de ampliar el corpus de definiciones incorporando evidencias provenientes de fuentes secundarias y terciarias, encontrar un método efectivo para probar el alcance y claridad de las definiciones hechas, así como resulta interesante plantearnos la relación entre la propuesta de definiciones y la forma en la que se establecerán los descriptores de las bases de datos, casos que se contemplan para futuras líneas de investigación del proyecto.

\section{Referencias}

1. Aguilar, C. A. (2008). Análisis lingüístico de definiciones en contextos definitorios. México: Universidad Nacional Autónoma de México.

2. Alfaro Asins, C., Marcos Alonso, C., Otero Morán, P. y Grañeda Miñón, P. (2009). Diccionario de numismática. Madrid: Ministerio de Educación, Cultura y Deporte.

3. Azevedo Delvizio, I. (2018). Directrices para la elaboración de definiciones terminológicas: una aplicación a los términos del turismo. Revista Digital Internacional de Lexicología, Lexicografía y Terminología, 1. Recuperado de: https://revistas.unc.edu.ar/index.php/ReDILLeT/article/view/22220/2 $\underline{1820}$

4. Baca Ibarra, I., Sierra Martínez, G. E., Aguilar, C. A., y Barceló, A. (2006). Estudio del modelo aristotélico aplicado al análisis y clasificación de definiciones en corpus lingüísticos especializados. Corpus, 1(1), pp. 1-10.

5. Bergenholtz, H., y Nielsen, S. (2013). The treatment of culture-bound items in dictionaries. En Gouws, R., Heid, U., Schweickard, W. y 
Wiegand, H. E. (Eds.), Dictionaries. An International Encyclopedia of Lexicography: Supplementary Volume: Recent Developments with Focus on Electronic and Computational Lexicography (pp. 469-481). Berlín: Walter de Gruyter.

6. Bergenholtz, H., y Tarp, S. (1995). Manual of specialised lexicography. Amsterdam/Philadelphia: John Benjamins Publishing Company. DOI: https://doi.org/10.1075/btl.12

7. Cabré i Castellví, M. T. (1999). Terminología y Lingüística: la teoría de las puertas. Estudios de Linguistica Del Español, 16. Recuperado de: http://elies.rediris.es/elies16/Cabre.html

8. Casal, J., y Mateu, E. (2003). Tipos de Muestreo. Revista de Epidemiología y Medicina Preventiva, 1, pp. 3-7.

9. Dubuc, R. (1999). Manual práctico de terminología. Santiago: Unión latina/RIL editores.

10. García de Quesada, M., y Montero Martínez, S. (2003). Hacia una gramática de la definición terminográfica. En Gallardo San Salvador, N. (Ed.), Terminología y traducción: Un bosquejo de su evolución (pp. 243-254). Granada: Editorial Atrio.

11. Haensch, G., y Lothar, W. (1982). La lexicografía. De la lingüística teórica a la lexicografía práctica. Madrid: Gredos.

12. Kipfer, B. A. (2013). Glossary of lexicographic terms. En Jackson, H. (Ed.), The Bloomsbury Companion to Lexicography (pp. 391-406). Londres/Nueva Delhi/Nueva York/Sidney: Bloomsbury Publishing.

13. Lara Ramos, L. F. (1994). Tipos de definición lexicográfica en el Diccionario del Español de México. In Garza Cuarón, B, Pascual, J. A. y Alonso González, A. (Eds.), Actas del II Encuentro de Filólogos y Lingüistas de España y México (pp. 153-164). Salamanca:

Universidad de Salamanca/Junta de Castilla y León, Consejería de Cultura y Turismo.

14. Lara Ramos, L. F. (1996). Por una redefinición de la lexicografía hispánica. Nueva Revista de Filología Hispánica, XLIV(2), pp. 346364. DOI: https://doi.org/10.24201/nrfh.v44i2.1942

15. Lara Ramos, L. F. (1997). Teoría del diccionario monolingüe. México, D.F.: El Colegio de México.

16. Luna Traill, E., Vigueras Ávila, A., y Baez Pinal, G. E. (2005). Diccionario básico de lingüística. Mexico, D.F.: Universidad Nacional Autónoma de México/Instituto de Investigaciones Filológicas: Centro de Lingüística Hispánica.

17. Martínez de Sousa, J. (2009). Manual básico de lexicografía. Gijón: Ediciones Trea.

18. Padilla, C., Maicas, R., y Cabrera, P. (2002). Diccionario de materiales cerámicos. Madrid: Ministerio de Educación, Cultura y Deporte.

19. Porto Dapena, J. Á. (2002). Manual de técnica lexicográfica. Madrid: Arco Libros.

20. Real Academia Española. (1726). Diccionario de la lengua castellana, en que se explica el verdadero sentido de las voces, su naturaleza y calidad, con las phrases o modos de hablar, los proverbios o refranes, y otras cosas convenientes al uso de la lengua [...]. Compuesto por la RAE. Madrid: Imprenta de Francisco del Hierro.

21. Rey-Debove, J. (1999). La linguistique du signe: une approche 
sémiotique du langage. Paris: Armand Colin.

22. Seminario de Música en la Nueva España y el México

Independiente. (2009). MUSICAT - Seminario de música en la Nueva España y el México independiente. Recuperado de: http://musicat.unam.mx/

23. Valero Doménech, E. (2007). Análisis de las definiciones y elaboración de un patrón definitorio del grupo de términos 'defectos del producto cerámico'. Barcelona: Universitat Jaume I.

24. Valero Doménech, E., y Alcina Caudet, M. (2015). Aspectos críticos de la formalización de características conceptuales en la definición terminográfica. Terminàlia, 11(11), pp. 30-44. DOI: https://doi.org/10.2436/20.2503.01.75

25. Wikimedia Commons. (2019). Wikipedia, la enciclopedia libre. Wikipedia. Recuperado de: https://es.wikipedia.org/wiki/Wikipedia:Portada

26. Wüster, E. (1998). Introducción a la teoría general de la terminología y a la lexicología terminológica. Barcelona: Institut Universitari de Lingüística Aplicada. Universitat Pompeu Fabra.

\section{Anexo. Guía rápida para la elaboración de definiciones}

a. Definiciones cortas. Son las que aparecerán en el globo azul en la base de datos y funcionan tanto para términos como para arcaísmos. Constan de dos partes:

1. Genus o género. Puede ser una unidad léxica (palabra o término) o una frase que identifique el tipo o la clase a la que esta unidad léxica pertenece. Para establecerlo es útil completar esta oración: "El (poner aquí al definido) es un tipo o clase de...

Ejemplos:

El cantor es un tipo o clase de cargo u oficio.

El bajón es un tipo o clase de instrumento musical.

2. Differentia o diferencia específica. Califica o caracteriza a la unidad léxica definida; tiene la función de distinguirla de otros conceptos de la misma clase que se encuentran en relación jerárquica horizontal. Generalmente se liga con el genus por medio de las formas léxicas: "que, cuyo, de quien, cuál, entre otras, o, incluso, de cualquier participio". Ejemplo: capellán de coro. Cargo u oficio de quien encabezaba el canto litúrgico a cambio de un pago fijo.

b. Definiciones largas. Se integrarán al glosario de términos, únicamente. Estas definiciones, además del genus y la differentia (definición analítica), contienen otros elementos que la complementan, a saber:

1. Definición funcional. Da cuenta de la función que desempeña la persona o la finalidad de un objeto que se define. Se puede establecer usando fórmulas como "que tiene la función", "que sirve para" o cualquier otra estructura semejante.

4. Definición extensional. Enumera elementos que componen al concepto. Se puede introducir mediante una enumeración simple o una enumeración de enumeraciones utilizando las convenciones ortográficas del español relativas al uso de la coma (,); y el punto y coma (;) y la coma (,), en combinación.

5. Definición enciclopédica. Amplía la información sobre el concepto aportando datos enciclopédicos o de relevancia para complementar el conocimiento del contexto cultural en el que este se usa o usó.

6. Definición sinonímica. Da cuenta de otros arcaísmos o términos que funcionan como sinónimos del que se define. Se recomienda usar una fórmula como "También es conocido como...".

Las definiciones se podrían ver así:

Ejemplo del término "capellán de coro"

Capellán de coro. Cargo u oficio de quien, en las catedrales, encabezaba el canto litúrgico [def. analítica]. Estos tenían la función de cantar canto llano en las misas y principales Horas Canónicas de los días festivos, y en otros eventos que estableciera el cabildo [def. funcional]. En 
los Estatutos de Erección de la Catedral Metropolitana de México, que fueron el modelo para las demás catedrales novohispanas, se instituían seis capellanes -llamados en la Catedral Metropolitana capellanes de Erección-, aunque su número varió en función de las necesidades y recursos de cada catedral. En cada catedral hubo, además, otros capellanes con obligación de cantar en el coro, como los capellanes de Lorenzana en la Catedral de México, los de Hevia y de la Virgen en Durango, o los de Don Vasco en Valladolid de Michoacán [def. enciclopédica].

Nota: no todas las definiciones incluyen, forzosamente, todos los tipos enumerados anteriormente. La inclusión o no de ellos queda a criterio del redactor y las definiciones serán revisadas en las reuniones del seminario, previo a su publicación. 